education and were behaviorally impaired. (Autti-Ramo I. Dev Med Child Neurol June 2000;42:406-411). Follow-up should be focussed not only on the child's cognitive and neuromotor development but on parental and foster family support and counseling and the prevention of secondary behavioral problems.

\title{
AMPHETAMINE ABUSE AND INTRACRANIAL HEMORRHAGE
}

Eight young adults presenting with intracranial hemorrhage at the Department of Neurosurgery, Queen's Medical Center and University Hospital, Nottingham, UK, in a 3 and $1 / 2$ year period, were diagnosed with amphetamine abuse. The time from amphetamine exposure to onset of symptoms ranged from 10 minutes to 2 months (median within 24 hours). CT and cerebral digital subtraction angiography (DSA) showed a parenchymal hematoma in 7 (3 in the frontal lobe), and a subarachnoid hemorrhage in 1 . Beading of small and medium-sized arteries, characteristic of vasculitis, also occurred in 1 . Four recovered, but 1 died and 3 were hemiplegic.

Of 37 previously reported cases, from 1945 to 1996,6 died and 17 had hemiparesis. One-third claimed to be infrequent amphetamine users. The first reported death from amphetamine abuse (IAMA 1939) was in a 25 year-old man who had taken the drug as a stimulant before a college examination. Amphetamine use or abuse should be considered in diagnosis of a frontal or parietal lobe hematoma in a young patient. (Buxton N, McConachie NS. Amphetamine abuse and intracranial hemorrhage. IR Soc Med September 2000;93:472-477). (Respond: Mr Neil Buxton FRCS (Ed), Department of Neurosurgery, University Hospital, Queen's Medical Centre, Nottingham NG7 2UH, UK).

COMMENT. Amphetamines taken orally, IV, or rarely, inhaled, have resulted in cerebral vasculitis and intracranial hemorrhage. Unfortunately, the dosages involved are unknown. The outcome is poor in more than half the cases reported. Vasculitis, that can follow only a single exposure to oral amphetamines, is characterized by irregular segmental narrowing or beading of small cerebral arteries.

Perhaps the recent increased popularity of amphetamine drugs in the treatment of ADHD in school-age children should be reappraised.

\section{CELIAC ANTIBODIES AND NEUROLOGIC DISORDERS}

The yield of screening for celiac disease in children with common neurologic disorders was evaluated at Tel Aviv University, Zerifin, Israel. Of 167 patients presenting, ages 1 to 16 years, 41 had migraine headaches, 39 ADHD, 36 epilepsy, and 51 hypotonia and motor abnormalities. IgG antigliadin antibodies were positive in 22 (13\%) of patients compared to $3(9 \%)$ in the control group. IgA and endomysial antibodies were negative in all patients, and duodenal biopsies were not performed. Routine screening for celiac disease is not recommended in children presenting wsith common neurologic disorders. (Lahat E, Broide E, Leshem M, Evans S, Scapa E. Prevalence of celiac antibodies in children with neurologic disorders. Pediatr Neurol May 2000;22:393-396). (Respond: Dr Eli Lahat, Unit of Pediatric Neurology, Assaf Harofeh Medical Center, Zerifin 70300, Israel).

COMMON. Neurologic complications of celiac disease include peripheral neuropathy, myopathy, cerebellar ataxia, myoclonus, cerebral atrophy, cerebral vasculitis, encephalitis, epilepsy, sometimes associated with cerebral calcification. Studies in adults have shown a significant increase in positive titers for antigliadin antibodies in patients (57\%) with common neurologic disorders of undetermined etiology. In children, the association of celiac disease with common 